\title{
Weak Riemannian manifolds from finite index subfactors*
}

\author{
Esteban Andruchow and Gabriel Larotonda
}

\begin{abstract}
Let $N \subset M$ be a finite Jones' index inclusion of $\mathrm{II}_{1}$ factors, and denote by $U_{N} \subset U_{M}$ their unitary groups. In this paper we study the homogeneous space $U_{M} / U_{N}$, which is a (infinite dimensional) differentiable manifold, diffeomorphic to the orbit

$$
\mathcal{O}(p)=\left\{u p u^{*}: u \in U_{M}\right\}
$$

of the Jones projection $p$ of the inclusion. We endow $\mathcal{O}(p)$ with a Riemannian metric, by means of the trace on each tangent space. These are pre-Hilbert spaces (the tangent spaces are not complete), therefore $\mathcal{O}(p)$ is a weak Riemannian manifold. We show that $\mathcal{O}(p)$ enjoys certain properties similar to classic Hilbert-Riemann manifolds. Among them, metric completeness of the geodesic distance, uniqueness of geodesics of the Levi-Civita connection as minimal curves, and partial results on the existence of minimal geodesics. For instance, around each point $p_{1}$ of $\mathcal{O}(p)$, there is a ball $\left\{q \in \mathcal{O}(p):\left\|q-p_{1}\right\|<r\right\}$ (of uniform radius $r$ ) of the usual norm of $M$, such that any point $p_{2}$ in the ball is joined to $p_{1}$ by a unique geodesic, which is shorter than any other piecewise smooth curve lying inside this ball. We also give an intrinsic (algebraic) characterization of the directions of degeneracy of the submanifold inclusion $\mathcal{O}(p) \subset \mathcal{P}\left(M_{1}\right)$, where the last set denotes the Grassmann manifold of the von Neumann algebra generated by $M$ and $p 1$
\end{abstract}

\section{Introduction}

Let $N \subset M$ be a finite index inclusion of $\mathrm{II}_{1}$ factors, and let $U_{N} \subset U_{M}$ be their unitary groups. In this paper we study the homogeneous space $U_{M} / U_{N}$ as an example of a weak Riemannian manifold, i.e. an infinite dimensional manifold with a Riemannian metric which makes the tangent spaces pre-Hilbert spaces. This paper is a continuation of [5], where the basic topological and differential facts of this space were established. Mainly, that there exists a concrete model for this homogeneous space, which is diffeomorphic to it. Let us describe this model. Consider Jones' basic construction: let $\mathrm{p}$ denote the Jones projection of the inclusion, i.e. the orthogonal projection

$$
\mathrm{p}: L^{2}(M, \tau) \rightarrow L^{2}(N, \tau)
$$

where $L^{2}(M, \tau)$ and $L^{2}(N, \tau)$ are the GNS Hilbert spaces of the trace $\tau$ (we denote indistinctly by $\tau$ the traces of $N$ and $M$ ). Consider the von Neumann algebra $M_{1}$ of operators in $L^{2}(M, \tau)$ generated by $M$ and $\mathrm{p}$. Then

$$
U_{M} / U_{N} \simeq \mathcal{O}(\mathrm{p}):=\left\{u \mathrm{p} u^{*}: u \in U_{M}\right\} \subset M_{1} .
$$

\footnotetext{
*2000 MSC. Primary 58B20; Secondary 46L10, 53C30, 53C22.

${ }^{1}$ Keywords and phrases: homogeneous space, short geodesic, Levi-Civita connection, Riemannian submanifold, totally geodesic submanifold, finite index inclusion, von Neumann $I I_{1}$ subfactor, Jones' projection, trace quadratic norm.
} 
This natural bijection is a homeomorphism. In [5] it was shown that this orbit $\mathcal{O}(\mathrm{p})$ is a submanifold of the Grassmann manifold $\mathcal{P}\left(M_{1}\right)$ (=selfadjoint projections) of $M_{1}$. Moreover, this regularity property in fact is equivalent to the finite index condition. The Grassmann manifold of a $C^{*}$-algebra is a well behaved Finsler manifold, with a linear connection coming from a reductive structure, and the Finsler metric induced by the usual norm of the algebra 8 , 15. The geodesics of the linear connection are minimal curves between points (=projections) lying at norm distance less than 1.

It is well known [9, 14] that $M_{1}$ is also a type $\mathrm{II}_{1}$ factor, and that the inclusion $M \subset M_{1}$ has the same index as the original inclusion. This gives rise the the so called basic construction, which we recall later. Denote also by $\tau$ be the trace of $M_{1}$ (which by restriction gives the traces of $M$ and $N)$.

In von Neumann algebras the relevant topologies are the weak topologies. The $\sigma$-strong, strong and weak operator topologies coincide in $\mathcal{P}\left(M_{1}\right)$. This set is a manifold only in the norm topology. Therefore, whenenever we use the word smooth, applied to a curve or a map, it is meant that is smooth in the norm structure. The weak topologies in $\mathcal{P}\left(M_{1}\right)$ are metrized by the 2 -norm induced by the trace, $\|x\|_{2}=\tau\left(x^{*} x\right)^{1 / 2}$. Although $\mathcal{P}\left(M_{1}\right)$ is not a submanifold of the GNS Hilbert space completion $L^{2}\left(M_{1}, \tau\right)$, it is a complete metric space, the geodesic metric is equivalent to the 2-metric, and the geodesics of the linear connection mentioned above behave nicely with respect to the 2 -norm (in fact, with the $k$-norms, for $k \geq 2$ ): if two projections lie at (norm) distance less than 1 , then the geodesic that joins them is shorter than any other piecewise smooth curve, when the length is measured with the 2-norm [3].

In this paper we study the metric induced in $\mathcal{O}(\mathrm{p})$ by the trace inner product $\langle x, y\rangle=$ $\tau\left(y^{*} x\right)$ at each tangent space. The tangent spaces

$$
(T \mathcal{O}(\mathrm{p}))_{q}=\left\{x q-q x: x \in M, x^{*}=-x\right\}
$$

are not complete with this metric. Therefore this study does not fit in the classical (infinite dimensional) Riemannian theory [10. Certain computations, though, can be carried out nicely, as in the classical case: e.g. the geodesics of the Levi-Civita connection can be explicitly computed. However, the fact that the tangent spaces are not complete allow for certain unusual phenomena. For instance, one cannot find normal neighborhoods around each point. Nevertheless certain facts do hold. Let us remark, for example, that $\mathcal{O}(\mathrm{p})$, with the geodesic distance induced by this incomplete metric, is in fact a complete metric space (Theorem 3.5). Also we prove (Theorem 5.2), that if there exists a curve that is piecewise smooth (in the norm structure), such that it has minimal length for the trace metric, then it is a geodesic of the linear connection. As for existence of minimal geodesics, we show (Theorem 5.3) that there exists a radius $r$, such that if $\left\|p_{1}-p_{2}\right\|<r, p_{1}, p_{2} \in \mathcal{O}(\mathrm{p})$, then there exists a geodesic of the Levi-Civita connection of the trace, with $p_{1}$ and $p_{2}$ as its endpoints, which is shorter than any other piecewise smooth curve lying inside the ball of center $p_{1}$ and radius $r$. Regarding the submanifold inclusion $\mathcal{O}(\mathrm{p}) \subset \mathcal{P}\left(M_{1}\right)$ (see [5]), we show (Theorem 6.3) that the Riemannian curvature of this inclusion is related to the inclusion $\left\{x^{*}=-x \in \operatorname{ker} E: x^{2} \in N\right\} \subset M$, and this set is exactly the set of directions of degeneracy in $\mathcal{O}(\mathrm{p})$ (i.e. the directions $x$ in the tangent bundle such that the geodesic of $\mathcal{P}\left(M_{1}\right)$ with initial speed $x$ is also a geodesic of $\left.\mathcal{O}(\mathrm{p})\right)$.

The contents of the paper are as follows. In Section 2 we recall certain basic facts, as the Jones basic construction, and establish topologic properties of $\mathcal{O}(\mathrm{p})$, both in the norm and weak topologies. In Section 3 we introduce the Riemannian structure in $\mathcal{O}(\mathrm{p})$ by means of the trace $\tau$. We show that though the tangent spaces are incomplete, the metric space $\mathcal{O}(\mathrm{p})$ with the Riemannian (or geodesic) distance is complete. Also we study the horizontal lifting of curves, and its metric properties. Based on a result on the local convex structure of the unitary group in the $k$-norms proved in [4, we prove that geodesics are short curves (in the 
2-metric) among curves which do not exceed certain length (measured in the usual norm Finsler metric). This result is the key fact to our minimality results in Section 5, where we also prove the uniqueness of geodesics as minimal curves. In Section 6 we study the geodesics of the Riemannian inclusion $\mathcal{O}(\mathrm{p}) \subset \mathcal{P}\left(M_{1}\right)$.

\section{Topological considerations}

Let $N \subset M$ be an inclusion of $\mathrm{II}_{1}$ factors with trace $\tau$, and finite index $[M: N]=\lambda^{-1}$. Let $L^{2}(M, \tau)$ and $L^{2}(N, \tau)$ be the GNS Hilbert spaces of $\tau$ and let $\mathrm{p}: L^{2}(M, \tau) \rightarrow L^{2}(N, \tau)$ be the (Jones) orthogonal projector. This projection induces the unique trace-preserving conditional expectation $E: M \rightarrow N$. Let $M_{1}$ be the von Neumann algebra of operators in $L^{2}(M, \tau)$ generated by $\mathrm{p}$ and $M$. This construction, known as the basic construction, enjoys several properties. Among them [9, 14]:

1. $M_{1}$ is a finite factor, $\left[M_{1}: M\right]=[M: N]=\lambda^{-1}$.

2. $\mathrm{p} x \mathrm{p}=E(x) \mathrm{p}$ for any $x \in M$.

3. $\{\mathrm{p}\}^{\prime} \cap M=N$.

4. $N \ni x \mapsto x \mathrm{p} \in N \mathrm{p}=\mathrm{p} M_{1} \mathrm{p}$ is a $*$-isomorphism.

5. $M_{1} \mathrm{p}=M \mathrm{p}$, and therefore $M \mathrm{p}$ is a closed linear subspace of $M_{1}$.

6. The $\operatorname{map} M \ni a \mapsto a \mathrm{p} \in M \mathrm{p}$ is a linear isomorphism, with $\|a\| \geq\|a \mathrm{p}\| \geq \sqrt{\lambda}\|a\|$.

7. If we denote by $E_{1}: M_{1} \rightarrow M$ the unique $\tau$-preserving conditional expectation, then $E_{1}(\mathrm{p})=\lambda$.

8. $E\left(x^{*} x\right) \geq \lambda x^{*} x$ for any $x \in M$

Definition 2.1. Let $\mathcal{O}(\mathrm{p})=\left\{u \mathrm{p} u^{*}: u \in U_{M}\right\}$ be the $U_{M}$-unitary orbit of $\mathrm{p}$, viewed as a subset of $M_{1}$. Since $\{\mathrm{p}\}^{\prime} \cap M=N, \mathcal{O}(\mathrm{p})$ can be identified with the quotient $U_{M} / U_{N}$ via the $\operatorname{map} \ell_{\mathrm{p}}: U_{M} \rightarrow M_{1}, \ell_{\mathrm{p}}(u)=u \mathrm{p} u^{*}$.

Note that $\mathcal{O}(\mathrm{p}) \simeq U_{M} / U_{N}$ is a topological isomorphism (for the norm induced topologies), and when the index is finite, it can be proved that $\ell_{p}$ is a fibration (see [5]), and admits smooth cross-sections. In that paper it was proved that $\mathcal{O}(\mathrm{p})$ is a smooth submanifold of $\mathcal{P}\left(M_{1}\right)$ (the set of projections of $M_{1}$ ) if and only if the index of the inclusion $N \subset M$ is finite. The following result is certainley well known, and shall be used later. Using this fact below, we may construct local cross sections for $\mathcal{O}(\mathrm{p})$, from local cross sections for $\mathcal{P}\left(M_{1}\right)$.

Lemma 2.2. Suppose that $\omega \in U_{M_{1}}$ satisfies that $\omega \mathrm{p} \omega^{*} \in \mathcal{O}(\mathrm{p})$. Then there exists $u \in U_{M}$ such that $u \mathrm{p}=\omega \mathrm{p}$. Moreover, if $\omega(t)$ is a smooth curve of unitaries in $M_{1}$ with the same property, then the curve $u(t)$ of unitaries in $M$ can also be chosen smooth.

Proof. By the facts 5 and 6 in the list of properties of the basic construction listed at the beginning of Section 2, there exists $m \in M$ such that $m \mathrm{p}=\omega \mathrm{p}$. We claim that $m$ is a unitary element. Note that $\omega \mathrm{p} \omega^{*}=\omega \mathrm{p}(\omega \mathrm{p})^{*}=m \mathrm{p} m^{*} \in \mathcal{O}(\mathrm{p})$. Therefore there exists a unitary element $v \in U_{M}$ such that $m \mathrm{p} m^{*}=v \mathrm{p} v^{*}$. Then $v^{*} m \mathrm{p} m^{*} v=\mathrm{p}$. If we multiply by $\mathrm{p}$ on both sides, we obtain

$$
\mathrm{p}=\mathrm{p} v^{*} m \mathrm{p} m^{*} v \mathrm{p}=E\left(v^{*} m\right) \mathrm{p} E\left(m^{*} v\right) \mathrm{p}=E\left(v^{*} m\right) E\left(m^{*} v\right) \mathrm{p}
$$

because $\mathrm{p}$ commutes with $N$, and then by further properties of the basic construction,

$$
E\left(v^{*} m\right) E\left(m^{*} v\right)=E\left(v^{*} m\right) \mathrm{p} E\left(v^{*} m\right)^{*} \mathrm{p}=1 .
$$


Since $N$ is finite, this implies that $E\left(v^{*} m\right)$ is a unitary element. Then $\mathrm{p}=v^{*} m \mathrm{p} m m^{*} v \mathrm{p}=$ $v^{*} m E\left(m^{*} v\right) \mathrm{p}$, or equivalently, using that $E\left(v^{*} m\right)$ is unitary,

$$
v^{*} m \mathrm{p}=E\left(v^{*} m\right) \mathrm{p} .
$$

By property 6 of the list, this implies that $v^{*} m=E\left(v^{*} m\right)$, and therefore $m \in U_{M}$. If $\omega(t)$ is a smooth curve in $U_{M_{1}}$ with $\omega(t) \mathrm{p} \omega^{*}(t) \in \mathcal{O}(\mathrm{p})$, one proves that $u(t) \in U_{M}$ is also smooth, because $m$ above is obtained by composition of the map $M_{1} \ni \omega \mapsto \omega \mathrm{p} \in M \mathrm{p}$ with the inverse linear isomorphism of property $6, M \ni a \mapsto a \mathrm{p} \in M \mathrm{p}$.

This lemma above shows that $u$ can be computed by

$$
u=\frac{1}{\lambda} E_{1}(w \mathrm{p}) .
$$

It is known [3] that the Grassmann manifold $\mathcal{P}\left(M_{1}\right)$ has geodesical radius 1: two projections $p_{1}$ and $p_{2}$ in $M_{1}$ such that $\left\|p_{1}-p_{2}\right\|<1$ are joined by a unique curve $\gamma(t)=e^{t x} p_{1} e^{-t x}$, with $x^{*}=-x$ in $M_{1}, x p_{1}$-codiagonal, and $\|x\|<\pi / 2$. The condition that $x$ is $p_{1}$-codiagonal describes precisely that $\gamma$ is a geodesic of the connection in $\mathcal{P}\left(M_{1}\right)$, and means that $p_{1} x p_{1}=$ $\left(1-p_{1}\right) x\left(1-p_{1}\right)=0$, or equivalently, that $x=x p_{1}+p_{1} x$. In particular one may define a distinguished exponential local cross section for the map

$$
U_{M_{1}} \rightarrow \mathcal{P}\left(M_{1}\right), w \mapsto w p_{1} w^{*},
$$

namely

$$
s_{p_{1}}:\left\{p_{2} \in \mathcal{P}\left(M_{1}\right):\left\|p_{2}-p_{1}\right\|<1\right\} \rightarrow U_{M_{1}}, \quad s_{p_{1}}\left(p_{2}\right)=e^{x} .
$$

Putting these two facts together enables us to obtain that if $q \in \mathcal{O}(\mathrm{p})$ such that $\|q-\mathrm{p}\|<1$, then

$$
\theta_{\mathrm{p}}(q)=\frac{1}{\lambda} E_{1}\left(s_{\mathrm{p}}(q) \mathrm{p}\right),
$$

defines a real analytic (in the norm structure) local cross section for the homogeneous space $\mathcal{O}(\mathrm{p})$ :

$$
\theta_{\mathrm{p}}(q) \in U_{M} \quad \text { and } \quad \theta_{\mathrm{p}}(q) \mathrm{p} \theta_{\mathrm{p}}(q)^{*}=q .
$$

Our next result states that the identification $\mathcal{O}(\mathrm{p}) \simeq U_{M} / U_{N}$ also works in the strong operator topology. Note that since $N \subset M$ are finite, both $U_{M}, U_{N}$ are complete topological groups in the strong operator topology, which is metrized by the 2-norm \|\|$_{2}$. To prove our statement, we shall need the following result, which is certainly not unknown to specialists (see Lemma 5.3 in [6] for a proof):

Lemma 2.3. Let $N$ be a finite von Neumann algebra and let $a_{n} \in N$ such that $\left\|a_{n}\right\| \leq 1$ and $a_{n}^{*} a_{n} \rightarrow 1$ strongly. Then there exist unitaries $v_{n} \in N$ such that $a_{n}-v_{n} \rightarrow 0$ strongly.

Proposition 2.4. The natural bijection

$$
U_{M} / U_{N} \rightarrow \mathcal{O}(\mathrm{p}), \quad[u] \mapsto u \mathrm{p} u^{*}
$$

is a homeomorphism with the topologies induced by the strong operator topology.

Proof. Suppose that $\left\{\left[u_{d}\right]\right\}_{d \in D}$ is a net in the quotient $U_{M} / U_{N}$ which converges to $[u]$ in the topology induced by the strong operator topology. This implies that there exist unitaries $v_{\alpha} \in U_{N}$ such that $u_{\alpha} v_{\alpha} \rightarrow u$ strongly. Since $M$ is finite, this implies that also $v_{\alpha}^{*} u_{\alpha}^{*} \rightarrow u^{*}$ strongly. Then, using that multiplication is also strongly continuous,

$$
u_{\alpha} \mathrm{p} u_{\alpha}^{*}=u_{\alpha} v_{\alpha} \mathrm{p} v_{\alpha}^{*} u_{\alpha}^{*} \rightarrow u \mathrm{p} u^{*} .
$$


Note that $\mathcal{O}(\mathrm{p})$ is a bounded set in $M_{1}$, and therefore the strong topology is metrizable with the 2-norm. In particular we may replace all nets by sequences in our arguments. By the strong continuity of the action of $U_{M}$ on $\mathcal{O}(\mathrm{p})$ (again using that $M$ is finite), we only need to prove continuity of the inverse map at $\mathrm{p}$. Suppose that $u_{n} \mathrm{p} u_{n}^{*} \rightarrow \mathrm{p}$. Then we claim that $\left\|u_{n} E\left(u_{n}^{*}\right)-1\right\|_{2} \rightarrow 0$. Indeed

$$
\begin{aligned}
\left\|u_{n} E\left(u_{n}^{*}\right)-1\right\|_{2}^{2} & =\tau\left(E\left(u_{n}\right) E\left(u_{n}^{*}\right)\right)+1-\tau\left(u_{n} E\left(u_{n}^{*}\right)\right)-\tau\left(E\left(u_{n}\right) u_{n}^{*}\right) \\
& =1-\tau\left(E\left(u_{n}\right) E\left(u_{n}^{*}\right)\right),
\end{aligned}
$$

because $\tau\left(u_{n} E\left(u_{n}^{*}\right)\right)=\tau\left(E\left(u_{n} E\left(u_{n}^{*}\right)\right)\right)=\tau\left(E\left(u_{n}\right) E\left(u_{n}^{*}\right)\right)$ and similarly for the other term. Therefore it suffices to show that $\tau\left(E\left(u_{n}\right) E\left(u_{n}^{*}\right)\right) \rightarrow 1$ strongly. Note that $u_{n} \mathrm{p} u_{n}^{*} \rightarrow \mathrm{p}$ strongly implies that

$$
\mathrm{p} u_{n} \mathrm{p} u_{n}^{*} \mathrm{p}=E\left(u_{n}\right) E\left(u_{n}^{*}\right) \mathrm{p} \rightarrow \mathrm{p}
$$

strongly. Note that $E\left(u_{n}\right) E\left(u_{n}^{*}\right) \in N$ and recall that the map $N \rightarrow N$ p is a $*$-isomorphism, which is clearly normal. Therefore $E\left(u_{n}\right) E\left(u_{n}^{*}\right) \rightarrow 1$ strongly, and thus $\tau\left(E\left(u_{n}\right) E\left(u_{n}^{*}\right)\right) \rightarrow 1$. We may apply Lemma 2.3 to the elements $a_{n}=E\left(u_{n}^{*}\right)$. It follows that there exist unitaries $v_{n}$ in $N$ such that $E\left(u_{n}^{*}\right)-v_{n} \rightarrow 0$ strongly, and therefore $u_{n} v_{n}-u_{n} E\left(u_{n}^{*}\right) \rightarrow 0$ strongly. Thus

$$
u_{n} v_{n} \rightarrow 1
$$

which completes the proof.

Proposition 2.5. The orbit $\mathcal{O}(\mathrm{p})$ is a complete metric space in the 2-norm.

Proof. Suppose that $\left\{p_{n}\right\}$ is a Cauchy sequence in $\mathcal{O}(\mathrm{p})$, with $p_{n}=u_{n} \mathrm{p} u_{n}^{*}, u_{n} \in U_{M}$. Then $p_{n}$ converges strongly to an operator $q$ acting in $L^{2}\left(M_{1}, \tau\right)$. Indeed, for each $\eta \in L^{2}\left(M_{1}, \tau\right)$, $\left\{p_{n} \eta\right\}$ is a Cauchy sequence in $L^{2}\left(M_{1}, \tau\right)$. Let us prove this fact. If $\eta=x \in M \subset L^{2}\left(M_{1}, \tau\right)$,

$$
\begin{aligned}
\left\|p_{n} x-p_{k} x\right\|_{2}^{2} & =\tau\left(x^{*}\left(p_{n}-p_{k}\right)^{2} x\right)=\tau\left(\left(p_{n}-p_{k}\right) x^{*} x\left(p_{n}-p_{k}\right)\right) \\
& \leq\|x\|^{2} \tau\left(\left(p_{n}-p_{k}\right)^{2}\right)=\|x\|^{2}\left\|p_{n}-p_{k}\right\|_{2}^{2} .
\end{aligned}
$$

In general, there exists $x \in M$ such that $\|x-\eta\|_{2}<\epsilon / 2$. And therefore

$$
\begin{aligned}
\left\|\left(p_{n}-p_{k}\right) \eta\right\|_{2} & \leq\left\|\left(p_{n}-p_{k}\right) x\right\|_{2}+\left\|\left(p_{n}-p_{k}\right)(\eta-x)\right\|_{2} \\
& \leq\left\|\left(p_{n}-p_{k}\right) x\right\|_{2}+2\|\eta-x\|_{2}<\left\|\left(p_{n}-p_{k}\right) x\right\|_{2}+\epsilon .
\end{aligned}
$$

Therefore $p_{n}$ converges strongly to a linear operator in $\mathcal{H}$, which is bounded by the uniform boundedness principle, and lies in $M_{1}$, i.e. $p_{n} \rightarrow q \in M_{1}$. By strong continuity of the product and the adjoint $\left(M_{1}\right.$ is finite), clearly $q^{2}=q^{*}=q$. Moreover, since $\tau$ is normal and $\tau\left(p_{n}\right)=\tau(\mathrm{p})=\lambda$, it follows that $\tau(q)=\lambda$. Therefore $q=w \mathrm{p} w^{*}$ for $w \in U_{M_{1}}$, and thus by the properties of the basic construction, there exists $m \in M$ with $E\left(m^{*} m\right)=1$ such that $q=m \mathrm{p} m^{*}$. Using the conditional expectation $E_{M_{1}}: M_{1} \rightarrow M$, which verifies that $E_{M_{1}}(\mathrm{p})=\lambda$, one obtains that

$$
E_{M_{1}}\left(p_{n}\right)=u_{n} E_{M_{1}}(\mathrm{p}) u_{n}^{*}=\lambda,
$$

and therefore $E(q)=\lambda$. Then

$$
\lambda=E_{M_{1}}\left(m \mathrm{p} m^{*}\right)=m E_{M_{1}}(\mathrm{p}) m^{*}=\lambda m m^{*},
$$

that is, $m \in U_{M}$ and therefore $q \in \mathcal{O}(\mathrm{p})$. 
In a remarkable paper [16, S. Popa and M. Takesaki proved the following result [16, Lemma 3], based on the theory of continuous selections by E. Michael [13. Suppose that the separable $\mathrm{II}_{1}$ factor $N$ has the following property, which for brevity we will refer as the scaling property: the tensor product $N \otimes \mathcal{B}(H)$ ( $H$ a separable Hilbert space) admits a one parameter automorphism group $\left\{\theta_{s}: s \in \mathbb{R}\right\}$ scaling the trace of $N \otimes \mathcal{B}(H)$, i.e. $\tau \circ \theta_{s}=e^{-s} \tau$, $s \in \mathbb{R}$, with $\tau$ a faithful semi-finite normal trace in $N \otimes \mathcal{B}(H)$. Then $U_{N}$ is contractible in the strong operator topology, and moreover, if $M$ is another factor with $N \subset M$, then the quotient map

$$
U_{M} \rightarrow U_{M} / U_{N}
$$

admits a global cross section when these spaces are considered with the (topologies induced by) the strong operator topology. The family of algebras enjoying these scaling properties includes the hyperfinite factor $\mathcal{R}$. A straightforward consequence of this result follows:

Proposition 2.6. Suppose that $N$ has the scaling property. Then the map

$$
U_{M} \rightarrow \mathcal{O}(\mathrm{p}), \quad u \mapsto u \mathrm{p} u^{*}
$$

is a fibre bundle, with the topologies induced by the strong operator topology. If moreover also $M$ has the scaling property, then $\mathcal{O}(\mathrm{p})$ is contractible in the strong operator topology.

Proof. The proof follows using the homotopy exact sequence of the bundle above, noting that $\mathcal{O}(\mathrm{p}) \simeq U_{M} / U_{N}$ in the strong topology as well.

Let us finish this section with the following result, concerning the homotopic structure of $\mathcal{O}(\mathrm{p})$ in the norm topology, for general $N \subset M$.

Proposition 2.7. The space $U_{M} / U_{N}$ (hence also $\mathcal{O}(\mathrm{p})$ ) is simply connected in the norm induced topology.

Proof. In the norm topology, the map

$$
\ell_{\mathrm{p}}: U_{M} \rightarrow \mathcal{O}(\mathrm{p}), \quad \ell_{\mathrm{p}}(u)=u \mathrm{p} u^{*}
$$

is a fibre bundle with fibre $U_{N}$. It therefore induces an exact sequence for the homotopy groups

$$
\ldots \rightarrow \pi_{1}\left(U_{N}\right) \stackrel{T}{\rightarrow} \pi_{1}\left(U_{M}\right) \rightarrow \pi_{1}(\mathcal{O}(\mathrm{p})) \rightarrow \pi_{0}\left(U_{N}\right) \rightarrow \cdots
$$

H. Araki, M.B. Smith and L. Smith proved in [7 that $\pi_{1}\left(U_{N}\right)=\pi_{1}\left(U_{M}\right) \simeq \mathbb{R}$. Moreover, they showed that this isomorphism is given as follows. Any given loop $\beta$ in $U_{M}$, with base point 1 , is homotopic to a concatenation of loops of the form $\alpha_{p}(t)=e^{2 \pi i t p}$, for $p$ a projection. In other words, these loops $\alpha_{p}$ generate $\pi_{1}\left(U_{M}\right)$. The isomorphism is given by the map which sends the homotopy class of $\alpha_{p}$ to the real number $\tau(p)$. Therefore, via this identification, the map $T$ above, restricted to this set of generators is given by the inclusion map

$$
\{\tau(p): p \in \mathcal{P}(N)\} \hookrightarrow\{\tau(q): q \in \mathcal{P}(M)\},
$$

which is surjective, because both set equal the unit interval. Hence $\pi_{1}(\mathcal{O}(p))=0$. 


\section{Riemannian structure of $\mathcal{O}(p)$}

Using the 2-norm \|\|$_{2}$ we can measure the length of curves in the standard fashion:

$$
L_{2}(\alpha)=\int_{0}^{1}\|\dot{\alpha}(t)\|_{2} d t .
$$

Let $M_{a h}=\left\{x \in M: x^{*}=-x\right\}, N_{a h}=\left\{x \in N: x^{*}=-x\right\}$. These spaces identify with the Banach-Lie algebras of $U_{M}$ and $U_{N}$ respectively (i.e. $U_{M}=\exp \left(M_{a h}\right)$ and $\left.U_{N}=\exp \left(N_{a h}\right)\right)$. Using that the map

$$
\ell_{q}: U_{M} \rightarrow \mathcal{O}(\mathrm{p}), \quad \ell_{q}(u)=u q u^{*}
$$

is a $\mathrm{C}^{\infty}$-submersion for $q \in \mathcal{O}(\mathrm{p})$, one can compute the tangent spaces of $\mathcal{O}(\mathrm{p})$ at any $q$. The differential of $\ell_{q}$ at 1 is given by

$$
\delta_{q}(x)=d\left(\ell_{q}\right)_{1}(x)=x q-q x, \quad x \in M_{a h}
$$

Therefore

$$
(T \mathcal{O}(\mathrm{p}))_{q}=\left\{x q-q x: x \in M_{a h}\right\} .
$$

We have an identification via the differential of the quotient map $U_{M} \mapsto U_{M} / U_{N}$

$$
\left(T U_{M} / U_{N}\right)_{\mathrm{p}} \simeq M_{a h} / N_{a h}
$$

Remark 3.1. Since $\tau \circ E=\tau$, if we put $\mathcal{H}=\operatorname{ker} E \cap M_{a h}$ we have

$$
M_{a h}=N_{a h} \oplus \mathcal{H}
$$

where $\mathcal{H}$ acts as an orthogonal supplement of $N_{a h}$ in $M_{a h}$ (relative to the inner product given by $\tau)$. Note that $\mathcal{H}$ is $A d\left(U_{N}\right)$-invariant, namely $u \mathcal{H} u^{*}=\mathcal{H}$ for any $u \in U_{N}$; let $\mathcal{H}_{q}=u \mathcal{H} u^{*}=A d_{u}(\mathcal{H})\left(q=u \mathrm{p} u^{*}, u \in U_{M}\right)$. Likewise, we will write $E_{q}$ to denote the translated conditional expectation $E_{q}=A d_{u} \circ E \circ A d_{u^{*}}$, which maps $M$ onto the von Neumann algebra $A d_{u}(N)$.

Therefore the maps $\delta_{q}$ give isomorphisms:

$$
\delta_{q}: \mathcal{H}_{q} \rightarrow(T \mathcal{O}(\mathrm{p}))_{q}
$$

Definition 3.2. Let $\kappa_{q}$ be the inverse of this linear map, namely $\kappa_{q}(z q-q z)=z$ for $z \in \mathcal{H}_{q}$, $\kappa_{q}:(T \mathcal{O}(\mathrm{p}))_{q} \rightarrow \mathcal{H}_{q}$. Equivalently, $\kappa_{q}(y)=z$ where $z$ is the only element of $\mathcal{H}_{q}=$ ker $E_{q}$ such that $\delta_{q}(z)=y$. We will omit the subindex when $q=p$, i.e. $\kappa_{\mathrm{p}}=\kappa, \delta_{\mathrm{p}}=\delta$.

Note that $\kappa_{q}$ is the $A d$-translation of $\kappa$ to the point $q=u \mathrm{p} u^{*} \in \mathcal{O}(\mathrm{p})$, namely

$$
\kappa_{q}(v)=A d_{u} \circ \kappa \circ A d_{u^{*}}(v)=u \kappa\left(u^{*} v u\right) u^{*}
$$

Lemma 3.3. The isomorphism $\delta_{q}: T_{\mathrm{p}} \mathcal{O}(\mathrm{p}) \simeq \mathcal{H}$ above is almost isometric, i.e if $q \in \mathcal{O}(\mathrm{p})$,

$$
\left\|\delta_{q}(z)\right\|_{2}=\sqrt{2 \lambda}\|z\|_{2}
$$

for any $z \in \mathcal{H}_{q}$.

Proof. We use the properties of $E$ and $\tau$ in a $\mathrm{II}_{1}$ factor with finite index $\lambda$ :

$$
\|z \mathrm{p}-\mathrm{p} z\|_{2}^{2}=2 \tau\left(\mathrm{p} z \mathrm{p} z-\mathrm{p} z^{2} \mathrm{p}\right)=-2 \tau\left(E\left(z^{2}\right) \mathrm{p}\right)=-2 \lambda \tau\left(E\left(z^{2}\right)\right)=-2 \lambda \tau\left(z^{2}\right)=2 \lambda\|z\|_{2}^{2}
$$

The identity now follows from the unitary invariance of the 2-norm. 
In order to compute the linear connection induced by the trace metric, one needs compute first the orthogonal projection from the ambient space, i.e. $M_{1 h}=\left\{x \in M_{1}: x^{*}=x\right\}$ onto the tangent spaces of $\mathcal{O}(\mathrm{p})$. Recall from the properties of the basic construction, that $M_{1} \mathrm{p}=M \mathrm{p}$ induces a map

$$
R: M_{1} \rightarrow M, \quad R(x)=m
$$

characterized as the unique element $m \in M$ such that $m \mathrm{p}=x \mathrm{p}$. This map can be computed in terms of $E_{1}: E_{1}(x \mathrm{p})=E_{1}(m \mathrm{p})=m \lambda$, i.e.

$$
R(x)=\frac{1}{\lambda} E_{1}(x \mathrm{p}) .
$$

Then we claim that the $\tau$-orthogonal projection

$$
\Pi_{\mathrm{p}}: M_{1 h} \rightarrow(T \mathcal{O}(\mathrm{p}))_{\mathrm{p}}=\left\{z \mathrm{p}-\mathrm{p} z: z \in M_{a h}\right\}
$$

is given by

$$
\Pi_{\mathrm{p}}(x)=\frac{1}{2}\left[R(x)-R(x)^{*}, \mathrm{p}\right],
$$

or equivalently

$$
\Pi_{\mathrm{p}}(x)=\frac{1}{2 \lambda}\left[E_{1}(x \mathrm{p}-\mathrm{p} x), \mathrm{p}\right],
$$

where $[$,$] is the usual commutator of operators. First note that \Pi_{p}$ projects onto $(T \mathcal{O}(p))_{p}$. Apparently it takes values in this linear subspace. If $z \mathrm{p}-\mathrm{p} z \in(T \mathcal{O}(\mathrm{p}))_{\mathrm{p}}(z \in \mathcal{H})$, then $(z \mathrm{p}-\mathrm{p} z) \mathrm{p}=z \mathrm{p}$ because $\mathrm{p} z \mathrm{p}=0$. Therefore $R(z \mathrm{p}-\mathrm{p} z)=z$ and

$$
\Pi_{\mathrm{p}}(z \mathrm{p}-\mathrm{p} z)=\frac{1}{2}\left[z-z^{*}, \mathrm{p}\right]=z \mathrm{p}-\mathrm{p} z .
$$

Finally, note that it is symmetric. Indeed, if $x, y \in M_{1} h$,

$$
\begin{aligned}
<\Pi_{\mathrm{p}}(x), y>= & \frac{1}{2 \lambda} \tau\left(\left[E_{1}(x \mathrm{p}-\mathrm{p} x), \mathrm{p}\right] y\right) \\
= & \frac{1}{2 \lambda}\left\{\tau\left(E_{1}(x \mathrm{p}) \mathrm{p} y\right)-\tau\left(E_{1}(\mathrm{p} x) \mathrm{p} y\right)+\tau\left(E_{1}(x \mathrm{p}) y \mathrm{p}\right)+\tau\left(E_{1}(\mathrm{p} x) y \mathrm{p}\right)\right\} \\
= & \frac{1}{2 \lambda}\left\{\tau\left(E_{1}(x \mathrm{p}) E_{1}(\mathrm{p} y)\right)-\tau\left(E_{1}(\mathrm{p} x) E_{1}(\mathrm{p} y)\right)\right. \\
& \left.+\tau\left(E_{1}(x \mathrm{p}) E_{1}(y \mathrm{p})\right)+\tau\left(E_{1}(\mathrm{p} x) E_{1}(y \mathrm{p})\right)\right\},
\end{aligned}
$$

which is clearly a symmetric expression in $x$ and $y$. In order to obtain the symmetric projections onto the other tangent spaces $(T \mathcal{O}(\mathrm{p}))_{q}, q \in \mathcal{O}(\mathrm{p})$ one translates $\Pi_{\mathrm{p}}$ covariantly via the action of $U_{M}$, namely if $q=u \mathrm{p} u^{*}$ for $u \in U_{M}$

$$
\Pi_{q}: M_{1 h} \rightarrow(T \mathcal{O}(\mathrm{p}))_{q}, \quad \Pi_{q}=\operatorname{Ad}(u) \circ \Pi_{\mathrm{p}} \circ \operatorname{Ad}\left(u^{*}\right),
$$

which does not depend on the choice of $u$ by general reasons. Nevertheless note that

$$
\Pi_{q}(x)=\frac{1}{2 \lambda} u\left[E_{1}\left(u^{*} x u \mathrm{p}-\mathrm{p} u^{*} x u\right), \mathrm{p}\right] u^{*}=\frac{1}{2 \lambda}\left[E_{1}(x q-q x), q\right] .
$$

Remark 3.4. Let $X$ be a smooth vector field along a curve $\gamma \subset \mathcal{O}(p)$, then the covariant derivative induced by the trace inner product is given by

$$
\frac{D X}{d t}=\Pi_{\gamma}(\dot{X}) .
$$

We shall call this connection the Levi-Civita connection of $\mathcal{O}(\mathrm{p})$ for obvious reasons. 
This derivative is compatible with the metric and has no torsion, these facts follow from formal considerations (as in classical Riemannian geometry).

This derivative was introduced in [8] as the "spatial derivative" of the orbit (in a more general context). There it was given the expression

$$
\frac{D X}{d t}=\dot{X}+\frac{1}{2}\left\{\left[X, \kappa_{\gamma}(\dot{\gamma})\right]+\left[\dot{\gamma}, \kappa_{\gamma}(X)\right]\right\}
$$

which coincides with our expression above after routine calculations.

As already stated, $\mathcal{O}(\mathrm{p}) \subset \mathcal{P}\left(M_{1}\right)$ is a $C^{\infty}$ submanifold in the norm topology. In the strong topology (as metrized by \|\|$_{2}$ ) neither of these spaces are manifolds. Nevertheless, with the trace Riemannian metric, $\mathcal{P}\left(M_{1}\right)$ is well behaved: it is a complete metric space with both the Riemannian and the \|\|$_{2}$-metric, which are equivalent, and any pair of projections can be joined by minimal geodesics [3]. We do not know if the Riemannian metric and the \|\|$_{2}$-metric are equivalent in $\mathcal{O}(\mathrm{p})$. One has though the following inequality

$$
d_{M}\left(p_{1}, p_{2}\right) \geq\left\|p_{1}-p_{2}\right\|_{2},
$$

where $p_{1}, p_{2} \in \mathcal{O}(\mathrm{p})$ and $d_{M}$ denotes the Riemannian or geodesic distance. This can be proved regarding $\mathcal{O}(\mathrm{p})$ as a subset on $M_{1}$, and noting that any curve in $\mathcal{O}(\mathrm{p})$, as a curve in $M_{1}$, is longer than the straight line segment.

Using Proposition 2.5 one can prove the following:

Theorem 3.5. $\mathcal{O}(\mathrm{p})$ is a complete metric space with the Riemannian metric $d_{M}$.

Proof. Let $\left\{p_{n}\right\}$ be a Cauchy sequence in $\mathcal{O}(\mathrm{p})$ for the metric $d_{M}$, with $p_{n}=u_{n} \mathrm{p} u_{n}^{*}$. Then, by the above inequality, it is a Cauchy sequence for the 2-norm, and therefore there exists a projection $q$ such that $\left\|p_{n}-q\right\|_{2} \rightarrow 0$. Therefore, by 2.5, $q=u \mathrm{p} u^{*} \in \mathcal{O}(\mathrm{p})$. Conjugating with $u$, we may suppose without loss of generality that $q=\mathrm{p}$. Note that $\left\|p_{n}-\mathrm{p}\right\|_{2} \rightarrow 0$ is equivalent to $\left\|u_{n}-E\left(u_{n}\right)\right\|_{2} \rightarrow 0$, or $\tau\left(E\left(u_{n}\right)^{*} E\left(u_{n}\right)\right) \rightarrow 1$. Indeed

$$
\begin{aligned}
\left\|p_{n}-\mathrm{p}\right\|_{2}^{2} & =\left\|u_{n} \mathrm{p}-\mathrm{p} u_{n}\right\|_{2}^{2}=\tau(\mathrm{p})+\tau\left(u_{n}^{*} \mathrm{p} u_{n}\right)-\tau\left(\mathrm{p} u_{n}^{*} \mathrm{p} u_{n}\right)-\tau\left(u_{n}^{*} \mathrm{p} u_{n} \mathrm{p}\right) \\
& =2 \lambda-2 \tau\left(\mathrm{p} u_{n}^{*} \mathrm{p} u_{n} \mathrm{p}\right)=2 \lambda-2 \tau\left(E\left(u_{n}^{*}\right) E\left(u_{n}\right) \mathrm{p}\right)=2 \lambda\left(1-\tau\left(E\left(u_{n}^{*}\right) E\left(u_{n}\right)\right)\right) .
\end{aligned}
$$

Also note that, as in the proof of Proposition 2.4

$$
\left\|u_{n}-E\left(u_{n}\right)\right\|_{2}^{2}=1-\tau\left(E\left(u_{n}\right)^{*} E\left(u_{n}\right)\right) .
$$

Let us apply now Lemma 2.3 to the elements $a_{n}=E\left(u_{n}\right)$ in the algebra $N$, again, as in the proof of 2.4. Then there exist unitaries $v_{n}$ in $N$ such that $\left\|E\left(u_{n}\right)-v_{n}\right\|_{2} \rightarrow 0$. It follows that $\left\|u_{n}-v_{n}\right\|_{2} \rightarrow 0$, or equivalently,

$$
\left\|u_{n} v_{n}^{*}-1\right\|_{2} \rightarrow 0
$$

In Proposition 4.4 of [1, it was shown that in the unitary group of a finite von Neumann algebra $M$ with trace $\tau$, the 2-metric is equivalent to the geodesic distance induced by the 2-metric. Also in that paper, the minimal geodesics were characterized, as the exponentials $\delta(t)=e^{i t x}$ for $t \in[0,1]$, where $x^{*}=x$ and $\|x\| \leq \pi$. It follows that there exist $x_{n} \in M$ with $x_{n}^{*}=x_{n}$ and $\left\|x_{n}\right\| \leq \pi$ which achieve the geodesic distance between 1 and $u_{n} v_{n}^{*}=e^{i x_{n}}$. And by the above facts, if $\delta_{n}(t)=e^{i t x_{n}}, t \in[0,1]$, then

$$
L_{2}\left(\delta_{n}\right)=\left\|x_{n}\right\|_{2} \rightarrow 0 .
$$

Consider the curve

$$
\gamma_{n}(t)=e^{i t x_{n}} \mathrm{p} e^{-i t x_{n}} \in \mathcal{O}(\mathrm{p})
$$

which join $\mathrm{p}$ to $u_{n} v_{n}^{*} \mathrm{p} v_{n} u_{n}^{*}=u_{n} \mathrm{p} u_{n}^{*}$. Then

$$
d_{M}\left(p_{n}, \mathrm{p}\right) \leq L_{2}\left(\gamma_{n}\right)=\left\|x_{n} \mathrm{p}-\mathrm{p} x_{n}\right\|_{2} \leq 2\left\|x_{n}\right\|_{2} \rightarrow 0 .
$$


Remark 3.6. The geodesics of this connection that start at $q$ can be computed; they are the curves of the form $\alpha(t)=e^{t z} q e^{-t z}$, with $z \in \mathcal{H}_{q}$.

For any curve $\gamma \in \mathcal{O}(\mathrm{p})$ (not necessarily a geodesic) with $\gamma(0)=q$, there is an horizontal lifting $\Gamma$ to the unitary group $U_{M}$, which is characterized by the following properties (see [12])

1. $\ell_{q}(\Gamma)=\gamma$.

2. $\Gamma(0)=1$.

3. $\dot{\Gamma} \in \mathcal{H}_{\gamma} \Gamma$.

Moreover, $\Gamma$ is also characterized as the unique solution of the linear differential equation

$$
\left\{\begin{array}{l}
\dot{\Gamma}=\kappa_{\gamma}(\dot{\gamma}) \Gamma \\
\Gamma(0)=1
\end{array}\right.
$$

The following is an easy consequence of Lemma 3.3 above.

Proposition 3.7. Let $\gamma$ be a piecewise smooth curve in $\mathcal{O}(\mathrm{p})$, and let $\Gamma$ be its horizontal lifting. Then

$$
L_{2}(\gamma)=\sqrt{2 \lambda} L_{2}(\Gamma) .
$$

Proof. Since $\Gamma q \Gamma^{*}=\gamma$, then

$$
L_{2}(\gamma)=\int_{0}^{1}\|\dot{\gamma}\|_{2} d t=\int_{0}^{1}\left\|\dot{\Gamma} q \Gamma^{*}+\Gamma q \dot{\Gamma}^{*}\right\|_{2} d t=\int_{0}^{1}\left\|\Gamma\left(\Gamma^{*} \dot{\Gamma} q+q \dot{\Gamma}^{*} \Gamma\right) \Gamma^{*}\right\|_{2} d t .
$$

Since $\Gamma$ is a curve of unitaries, differentiating $\Gamma^{*} \Gamma=1$ one obtains that $\dot{\Gamma}^{*} \Gamma=-\Gamma^{*} \dot{\Gamma}$. Then the above integral equals

$$
\int_{0}^{1}\left\|\Gamma^{*} \dot{\Gamma} q-q \Gamma^{*} \dot{\Gamma}\right\|_{2} d t=\int_{0}^{1}\left\|\delta_{q}\left(\Gamma^{*} \dot{\Gamma}\right)\right\|_{2} d t=\sqrt{2 \lambda} \int_{0}^{1}\left\|\Gamma^{*} \dot{\Gamma}\right\|_{2} d t=\sqrt{2 \lambda} L_{2}(\Gamma) .
$$

This observation implies that one can compare the lengths of the horizontal liftings of the curves, instead of the curves themselves, and profit from the geodesic structure of the unitary group. This is the point of view adopted in this paper.

Let us finish this section proving that the horizontal lifting is the shortest possible lifting for a curve in $\mathcal{O}(\mathrm{p})$.

Proposition 3.8. Let $\gamma(t), t \in[0,1]$ be a piecewise smooth curve in $\mathcal{O}(\mathrm{p})$. Suppose that $u(t) \in U_{M}$ is a piecewise smooth lifting of $\gamma$. Then the horizontal lifting $\Gamma$ of $\gamma$ is shorter than $u$ :

$$
L_{2}(\Gamma) \leq L_{2}(u)
$$

When using the usual operator norm, one has the estimate

$$
L_{\infty}(\Gamma) \leq 2 L_{\infty}(u)
$$

Proof. We may suppose without loss of generality that $\gamma$ starts at $\mathrm{p}$. Note that $\gamma=\Gamma \mathrm{p} \Gamma^{*}=$ $u \mathrm{p} u^{*}$. Then $u^{*} \Gamma \in\{\mathrm{p}\}^{\prime} \cap M=N$. Differentiating,

$$
\dot{u}^{*} \Gamma+u^{*} \dot{\Gamma} \in N .
$$

Note that $u^{*} \dot{\Gamma}=\left(u^{*} \Gamma\right)\left(\Gamma^{*} \dot{\Gamma}\right) \in N \cdot \mathcal{H} \subset \mathcal{H}$. It follows that if $P_{\mathcal{H}}=I-E$ denotes the $\tau$-orthogonal projection onto $\mathcal{H}$, one has

$$
P_{\mathcal{H}}\left(\dot{u}^{*} \Gamma\right)=-u^{*} \dot{\Gamma}
$$


Therefore

$$
\|\dot{\Gamma}\|_{2}=\left\|-u^{*} \dot{\Gamma}\right\|_{2}=\left\|P_{\mathcal{H}}\left(\dot{u}^{*} \Gamma\right)\right\|_{2} \leq\left\|\dot{u}^{*} \Gamma\right\|_{2}=\left\|\dot{u}^{*}\right\|_{2}=\|\dot{u}\|_{2} .
$$

Thus

$$
L_{2}(\Gamma)=\int_{0}^{1}\|\dot{\Gamma}\|_{2} d t \leq\|\dot{u}\|_{2} d t=L_{2}(u) .
$$

For the assertion corresponding $L_{\infty}$, we use the estimate

$$
\left\|P_{\mathcal{H}}(x)\right\|=\|x-E(x)\| \leq 2\|x\| .
$$

Remark 3.9. Using the argument above, one can prove that if $\gamma$ is a smooth curve in $\mathcal{O}(\mathrm{p})$, and $u$ is a smooth lifting of $\gamma$ such that $L_{2}(u)=L_{2}(\Gamma)$, then there exists a unitary element $v_{0}$ commuting with $\gamma(0)$ (for instance, if $\gamma(0)=\mathrm{p}$, then $v_{0} \in U_{N}$ ), such that

$$
u(t)=\Gamma(t) v_{0}, \quad t \in[0,1] .
$$

That is, $u$ is essentially the horizontal lifting. Indeed, in the proof above it is shown in fact that $\|\dot{\Gamma}(t)\|_{2} \leq\|\dot{u}(t)\|_{2}$. If $u$ is smooth, then $\|\dot{u}(t)\|_{2}$ is continuous in the parameter $t$. Then the inequality above and the hypothesis that $L_{2}(u)=\int_{0}^{1}\|\dot{u}(t)\|_{2} d t=\int_{0}^{1}\|\dot{\Gamma}(t)\|_{2} d t=L_{2}(\Gamma)$ imply the equality

$$
\|\dot{\Gamma}(t)\|_{2}=\|\dot{u}(t)\|_{2},
$$

for all $t$. Therefore $\dot{u}^{*} \Gamma=P_{\mathcal{H}}\left(\dot{u}^{*} \Gamma\right)=-u^{*} \dot{\Gamma}$, or equivalently

$$
0=\dot{u}^{*} \Gamma+u^{*} \dot{\Gamma}=u^{*} \Gamma,
$$

i.e. $u^{*}(t) \Gamma(t)=u^{*}(0) \Gamma(0)=v_{0}$ for all $t$, with $v_{0}$ commuting with $\gamma(0)$.

We may also compare the length of the horizontal lifting with the lengths of liftings in $U_{M_{1}}$. We shall need Lemma 2.2 from section 2.

Proposition 3.10. Let $\omega(t) \in U_{M_{1}}, t \in[0,1]$, be a piecewise smooth lifting of $\gamma(t) \in \mathcal{O}(\mathrm{p})$. Then

$$
L_{2}(\Gamma) \leq \frac{1}{\sqrt{\lambda}} L_{2}(\omega) .
$$

Proof. By the lemma, there exists a piecewise smooth curve $u(t) \in U_{M}$ such that $u(t) \mathrm{p}=$ $\omega(t) \mathrm{p}$. In particular $u$ is a lifting (in $U_{M}$ ) of $\gamma$, and therefore by the preceding proposition,

$$
L_{2}(\Gamma) \leq L_{2}(u)
$$

On the other hand, since for $a \in M$ we have $\|a\|_{2}=\frac{1}{\sqrt{\lambda}}\|a \mathrm{p}\|_{2}$, then

$$
L_{2}(u)=\frac{1}{\sqrt{\lambda}} L_{2}(u \mathrm{p})=\frac{1}{\sqrt{\lambda}} L_{2}(\omega \mathrm{p}) \leq \frac{1}{\sqrt{\lambda}} L_{2}(u),
$$

were the last assertion follows from the fact that $\|x y\|_{2} \leq\|x\|_{2}\|y\|$.

\section{Convexity properties of the unitary group}

Our argument on the minimality of geodesics in Section 5 needs certain facts concerning the geometry of the unitary group $U_{M}$, which were proved in [4. First recall that curves of the form $\delta(t)=u e^{i t x}$ (with $x^{*}=x$ and $\|x\| \leq \pi$ ) have minimal length for the $k$-norms, $k \geq 2$ 3]. Based on this fact, in Theorem 2.1 of [4] it was shown that if $F_{2}$ denotes the energy functional

$$
F_{2}(\gamma)=\int_{0}^{1}\|\dot{\gamma}\|_{2}^{2} d t=\int_{0}^{1} \tau\left(\dot{\gamma}^{*} \dot{\gamma}\right) d t,
$$


for $\gamma$ a piecewise smooth curve in $U_{M}$, and $\gamma_{s}(t)$ is a smooth variation of $\gamma$, i.e.

$$
\gamma_{s}(t) \in U_{M}, s \in(-r, r), \quad t \in[0,1], \quad \gamma_{0}=\gamma,
$$

then the first variation of the energy functional is

$$
\left.\frac{1}{2} \frac{d}{d s} F_{2}\left(\gamma_{s}\right)\right|_{s=0}=\left.\tau\left(x_{0} y_{0}\right)\right|_{t=0} ^{t=1}-\int_{0}^{1} \tau\left(\frac{d}{d t}\left[x_{0}\right] y_{0}\right) d t,
$$

where

$$
x_{s}(t)=\gamma_{s}(t)^{*} \frac{d}{d t} \gamma_{s}(t) \text { and } y_{s}(t)=\gamma_{s}(t)^{*} \frac{d}{d s} \gamma_{s}(t) .
$$

The main result in [4] states the following:

Proposition 4.1. (Theorem 4.5 of [4]) Let $u_{0}, u_{1}, u_{2} \in U_{M}$, such that $\left\|u_{i}-u_{j}\right\|<$ $\sqrt{2-\sqrt{2}}=r$. Let $\delta(t)=u_{1} e^{t z}$ be the minimal geodesic joining $u_{1}$ and $u_{2}$. Then $f(s)=$ $d_{k}\left(u_{0}, \delta(s)\right)^{k}\left(d_{k}=\right.$ geodesic distance induced by the $k$-norm $)$ is a convex function $(s \in[0,1])$, for $k$ an even integer.

We shall use this result for the case $k=2$.

Take $z \in \mathcal{H}$; the curve $\alpha(t)=e^{t z} \mathrm{p} e^{-t z}$ is a geodesic of the Levi-Civita connection of $\mathcal{O}(\mathrm{p})$, joining $\mathrm{p}$ with $q=e^{z} \mathrm{p} e^{-z}$. We have $\dot{\alpha}(t)=e^{t z}(z \mathrm{p}-\mathrm{p} z) e^{-t z}$ hence

$$
\|\dot{\alpha}(t)\|_{2}=\|z \mathrm{p}-\mathrm{p} z\|_{2}=\|\dot{\alpha}(0)\|_{2}
$$

and

$$
L_{2}(\alpha)=\int_{0}^{1}\|\dot{\alpha}(t)\|_{2} d t=\sqrt{2 \lambda}\|z\|_{2}
$$

by Lemma 3.3 above. The following estimates will prove useful. Recall that $\|$ \| denotes the usual operator norm of the von Neumann algebra $M_{1}$ :

Lemma 4.2. For any $z \in \mathcal{H}=\operatorname{ker} E \cap M_{a h}$,

$$
\left\|\delta_{\mathrm{p}}(z)\right\|=\|z \mathrm{p}-\mathrm{p} z\| \geq \sqrt{\lambda}\|z\| .
$$

Proof. Since $\mathrm{p} z \mathrm{p}=0$,

$$
\|z \mathrm{p}-\mathrm{p} z\|^{2}=\left\|(z \mathrm{p}-\mathrm{p} z)^{2}\right\|=\left\|z \mathrm{p} z+\mathrm{p} z^{2} \mathrm{p}\right\|=\left\|-z \mathrm{p} z-\mathrm{p} z^{2} \mathrm{p}\right\|
$$

Note that $-z \mathrm{p} z$ and $-\mathrm{p} z^{2} \mathrm{p}$ are both positive operators, and for $a, b \geq 0,\|a\| \leq\|a+b\|$ hence

$$
\|z \mathrm{p}-\mathrm{p} z\|^{2} \geq\left\|\mathrm{p} z^{2} \mathrm{p}\right\|=\left\|\mathrm{p} E\left(z^{2}\right)\right\|=\left\|E\left(z^{2}\right)\right\| \geq \lambda\|z\|^{2}
$$

by the definition of $E$ and the index properties.

Lemma 4.3. If $z \in \mathcal{H}$, with $\|z\|<\sqrt{\lambda}$, then

$$
\left\|e^{z} \mathrm{p} e^{-z}-\mathrm{p}\right\| \geq\|z\|(\sqrt{\lambda}-\|z\|) .
$$

Proof. Note that $\sqrt{\lambda}<\pi$. First, we rewrite the expression on the left:

$$
\begin{aligned}
\left\|e^{z} \mathrm{p} e^{-z}-\mathrm{p}\right\| & =\left\|e^{z} \mathrm{p}-\mathrm{p} e^{z}\right\|=\left\|\delta_{\mathrm{p}}(z)+\left(e^{z}-1-z\right) \mathrm{p}-\mathrm{p}\left(e^{z}-1-z\right)\right\| \\
& \geq\left\|\delta_{\mathrm{p}}(z)\right\|-\left\|\left(e^{z}-1-z\right) \mathrm{p}-\mathrm{p}\left(e^{Z}-1-z\right)\right\| \\
& \geq\left\|\delta_{\mathrm{p}}(z)\right\|-2\left\|e^{z}-1-z\right\| .
\end{aligned}
$$


Now, since $\sigma\left(e^{z}-1-z\right) \subset\left\{e^{i t}-1-i t:|t| \leq\|z\|\right\}$,

$$
\begin{aligned}
\left\|e^{z}-1-z\right\| & =\rho\left(e^{z}-1-z\right)=\sup _{|t| \leq\|z\|} \sqrt{(\cos (t)-1)^{2}+(\sin (t)-t)^{2}} \\
& =\sup _{|t| \leq\|z\|} \sqrt{2-2 \cos (t)-2 t \sin (t)+t^{2}} \\
& =\sqrt{2-2 \cos \|z\|-2\|z\| \sin \|z\|+\|z\|^{2}} .
\end{aligned}
$$

Using Taylor's series for the function under the square root, one can obtain the bound $\frac{1}{2}\|z\|^{2}$; hence

$$
\left\|e^{z} \mathrm{p} e^{-z}-\mathrm{p}\right\| \geq\left\|\delta_{\mathrm{p}}(z)\right\|-\|z\|^{2} .
$$

This bound together with the one in the previous lemma gives the desired inequality.

Denote by $L_{\infty}(\gamma)$ the length of the curve $\gamma$, measured in the norm metric of $\mathcal{O}(\mathrm{p})$, namely

$$
L_{\infty}(\gamma)=\int_{0}^{1}\|\dot{\gamma}(t)\| d t
$$

Lemma 4.4. If $\gamma$ is a smooth curve in $\mathcal{O}(\mathrm{p})$, and $\Gamma$ is the horizontal lift of $\gamma$, then

$$
\|\Gamma(1)-1\| \leq \frac{1}{\sqrt{\lambda}} L_{\infty}(\gamma)
$$

Proof. First note that

$$
\|\Gamma(1)-1\|=\left\|\int_{0}^{1} \Gamma \dot{\Gamma}(t) d t\right\| \leq \int_{0}^{1}\|\dot{\Gamma}(t)\| d t=\int_{0}^{1}\left\|\kappa_{\gamma}(\dot{\gamma})\right\| d t \leq \int_{0}^{1}\left\|\kappa_{\gamma}\right\|\|\dot{\gamma}\| d t .
$$

Now if $q \in \mathcal{O}(\mathrm{p}),\left\|\kappa_{q}\right\|=\left\|\kappa_{\mathrm{p}}\right\|$ because the action of the unitary group is isometric. Since $\kappa_{\mathrm{p}}=\delta_{\mathrm{p}}^{-1}$, where $\delta_{\mathrm{p}}: \mathcal{H} \rightarrow T_{\mathrm{p}} \mathcal{O}(\mathrm{p})$, by the bound in lemma 4.2, we have $\left\|\kappa_{\mathrm{p}}(v)\right\| \leq \frac{1}{\sqrt{\lambda}}\|v\|$, namely $\left\|\kappa_{q}\right\| \leq \frac{1}{\sqrt{\lambda}}$ for any $q \in \mathcal{O}(\mathrm{p})$. Hence

$$
\|\Gamma(1)-1\| \leq \frac{1}{\sqrt{\lambda}} \int_{0}^{1}\|\gamma(t)\| d t=\frac{1}{\sqrt{\lambda}} L_{\infty}(\gamma) .
$$

We say that a subset $V \subset U_{M}$ is geodesically convex if for any pair of elements $u_{0}, u_{1} \in V$ with $\left\|u_{0}-u_{1}\right\|<2$, the unique minimal geodesic of $U_{M}$ joining them lies in $V$.

Theorem 4.5. There exists a positive constant $R>0$ with the following property. Suppose that $q_{0}, q_{1} \in \mathcal{O}(\mathrm{p})$ with $q_{1}=e^{z} q_{0} e^{-z} \in \mathcal{O}(\mathrm{p})$ for $z \in \mathcal{H}_{q_{0}}$ with $\|z\| \leq R$, and let $\alpha(t)=$ $e^{t z} q_{0} e^{-t z}$ be the geodesic joining $q_{0}$ and $q_{1}$. If $\gamma$ is any other smooth curve joining $q_{0}$ and $q_{1}$, then either

$$
L_{\infty}(\gamma) \geq L_{\infty}(\alpha),
$$

or

$$
L_{2}(\gamma) \geq L_{2}(\alpha)
$$

Proof. Clearly all the norms involved are unitarily invariant, therefore we may suppose $q_{0}=\mathrm{p}$ without loss of generality. Let $\Gamma$ be the unitary lift of $\gamma$ in $U_{M}$. If $L_{\infty}(\gamma) \leq L_{\infty}(\alpha)$, by Lemma 4.4 above

$$
\|\Gamma(1)-1\| \leq \frac{1}{\sqrt{\lambda}}\|z \mathrm{p}-\mathrm{p} z\| \leq \frac{2}{\sqrt{\lambda}}\|z\| .
$$

Therefore we may adjust $R$ in order that $1, e^{z}$ and $\Gamma(1)$ stand in the situation where Proposition 4.1 applies, namely, that they lie closer than $\sqrt{2-\sqrt{2}}$ in norm. Let $\delta(s)=e^{z} e^{s w}$ be 
the unique minimizing geodesic joining $\delta(0)=e^{z}$ and $\delta(1)=e^{z} e^{w}=\Gamma(1)$ in $U_{M}$ (which is minimizing for all $k$-norms, and therefore in particular for the functional $L_{2}[3]$ ). Then by Proposition 4.1, the map

$$
f(s)=d_{2}^{2}(1, \delta(s)), \quad s \in[0,1]
$$

is convex. We claim that $f^{\prime}(0)=0$, so that $f$ has an absolute minimum at $s=0$. Indeed, note that

$$
\|\delta(s)-1\|=\left\|1-e^{s w}\right\| \leq\left\|1-e^{w}\right\|=\|1-\Gamma(1)\|<2 .
$$

Therefore the antihermitic logarithm

$$
\log :\left\{u \in U_{M}:\|u-1\|<2\right\} \rightarrow\left\{x \in M_{a h}:\|x\|<\pi\right\}
$$

is well defined. Let $\gamma_{s}(t)=e^{t \log (\delta(s))}$. Note that $\gamma_{s}(t)$ is a smooth variation of $\gamma_{0}=\alpha$. Also note that at each $s$ it consists of minimizing geodesics, because $\|\log (\delta(s))\|<\pi$. Then

$$
f(s)=L_{2}\left(\gamma_{s}\right)^{2}=\|\log (\delta(s))\|_{2}^{2} .
$$

Note also that $f(s)=F_{2}\left(\gamma_{s}\right)$. Then $f^{\prime}(0)$ can be computed using the first variation formula (31). In our case

$$
x_{s}=\gamma_{s}^{*} \frac{d}{d t} \gamma_{s}(t)=\log (\delta(s))
$$

is independent of $t$, and therefore (3) reduces to

$$
f^{\prime}(0)=2 \tau\left(z y_{0}(1)\right)-\tau\left(z y_{0}(0)\right) \text {. }
$$

Note that $\Gamma(1)$ and $e^{z}$ lie in the fibre of $q_{1}$ (both $\gamma=\Gamma \mathrm{p} \Gamma^{*}$ and $\alpha$ have the same endpoints), which is of the form $e^{z} U_{N}$. Clearly this set is geodesically convex [4, implying that $\delta(s)$ lies in this fibre, and therefore $\dot{\delta}(0) \in e^{z} N_{a h}$. At $t=0, \gamma_{s}(0)=1$ for all $s$, therefore $y_{s}(0)=0$. At $t=1, \gamma_{s}(1)=\delta(s)$, so that $\gamma_{s}(1)=\delta^{*}(s) \dot{\delta}(s)$. Then $y_{0}(1)=\delta^{*}(0) \dot{\delta}(0)=e^{-z} \dot{\delta}(0) \in N_{a h}$. Then $\tau\left(z y_{0}(0)\right)=0$ and

$$
\tau\left(z y_{0}(1)\right)=\tau\left(E\left(z y_{0}(1)\right)\right)=\tau\left(E(z) y_{0}(1)\right)=0
$$

because $E(z)=0$.

Our claim proved, it implies that if we denote $A(t)=e^{t z}$, and $B(t)=e^{t \log (\Gamma(1))}, t \in[0,1]$, then

$$
L_{2}(A) \leq L_{2}(B) .
$$

On the other hand, by the fact on minimality of curves in $U_{M}[3]$, one has that $L_{2}(B) \leq L_{2}(\Gamma)$. Therefore

$$
L_{2}(A) \leq L_{2}(\Gamma) .
$$

Multiplying both members by $\sqrt{2 \lambda}$ one gets

$$
L_{2}(\alpha)=\sqrt{2 \lambda} L_{2}(A) \leq \sqrt{2 \lambda} L_{2}(\Gamma)=L_{2}(\gamma) .
$$

Remark 4.6. Note that there exists a constant $R_{1}>0$ such that $\left\|q_{1}-q_{0}\right\|<R_{1}$ is equivalent to the existence of $z \in \mathcal{H}_{q_{0}}$ with $e^{z} q_{0} e^{-z}=q_{1}$ and $\|z\|<R$. Indeed, for the norm topology, due to the differential structure of $\mathcal{O}(p)$, the map

$$
\exp _{q_{0}}: \mathcal{H}_{q_{0}} \rightarrow \mathcal{O}(\mathrm{p}), \exp _{q_{0}}(z)=e^{z} q_{0} e^{-z}
$$

is a local diffeomorphism by the inverse function theorem.

Therefore the result above can be rephrased replacing the requirement $\|z\|<R$ by $\left\|q_{0}-q_{1}\right\|<$ $R_{1}$. 
The theorem above states that geodesics are short among curves which are a priori short in the norm Finsler metric. In the next section we show a more general minimality result, but let us state this sentence with more precision, for we shall need it later.

Corollary 4.7. There exists a numbre $R_{2}>0$ such that if $\gamma$ is a smooth curve with $L_{\infty}(\gamma) \leq$ $R_{2}$, then there exists a geodesic $\delta$ joining the same endpoints as $\gamma$, with $L_{2}(\delta) \leq L_{2}(\gamma)$.

Proof. Choose $R_{2}<R_{1}$ in order to assure the existence of $\delta$ : if $q, r$ are the endpoints of $\gamma$, then $\|q-r\| \leq L_{\infty}(\gamma) \leq R_{2}<R_{1}$ and Remark 4.6 applies. Then the proof follows as in the theorem above, adjusting $R_{2}$ additionally in order that

$$
\|\Gamma(1)-1\| \leq \frac{1}{\sqrt{\lambda}} L_{\infty}(\gamma) \leq \frac{R_{2}}{\sqrt{\lambda}}<\sqrt{2-\sqrt{2}}
$$

\section{Geodesics as unique minimal curves}

A continuous curve $\alpha$ is a piecewise smooth geodesic if it consists of a finite collection $\left\{\alpha_{i}\right\}$ of geodesic arcs glued together, or in other words, a polygonal path with geodesic edges. Let us start with a brief result:

Proposition 5.1. Let $\gamma \subset \mathcal{O}(\mathrm{p})$ be any smooth curve joining $q$ to $r$. Then there exists a continuous piecewise smooth geodesic $\alpha=\cup \alpha_{i}$ which joins $q$ and $r$ such that

$$
\sum L_{2}\left(\alpha_{i}\right) \leq L_{2}(\gamma)
$$

Proof. Suppose $\gamma$ is parametrized in the interval $[0,1]$. Then there exists a partition $0=$ $t_{0}<t_{1}<\ldots<t_{n}=1$ of $[0,1]$ such that $\left\|\gamma\left(t_{i}\right)-\gamma\left(t_{i-1}\right)\right\|<R_{1}$ for $i=1 \ldots n$ with $R_{1}$ as in Remark 4.6. Then there exist geodesics $\alpha_{i}$, minimizing for the functional length $L_{2}$, which join $\gamma\left(t_{i-1}\right)$ with $\gamma\left(t_{i}\right)$. Then clearly $\alpha=\cup \alpha_{i}$ is shorter for the 2-metric than $\gamma$.

Our main result on uniqueness of geodesics as minimal curves follows.

Theorem 5.2. Let $\gamma \subset \mathcal{O}(\mathrm{p})$ be a piecewise smooth curve which is short (i.e minimizing) for the 2-metric. Then $\gamma$ is a geodesic of the Riemannian connection of $\mathcal{O}(\mathrm{p})$.

Proof. By the previous lemma, there exists a piecewise smooth geodesic $\alpha=\cup \alpha_{i}$ which is shorter than $\gamma$. Then $\alpha$ is also minimizing (in fact, $L_{2}(\gamma)=\sum L_{2}\left(\alpha_{i}\right)=L_{2}(\alpha)$ ). Moreover, the elements $t_{0}, t_{1}, \ldots, t_{n}$ of the partition provide points $\gamma\left(t_{i}\right)$ which lie both in $\gamma$ and $\alpha$. We claim that $\alpha$ is smooth, i.e. a geodesic. This proves our result, since the partition can be arbitrarily refined to contain as many (finite) points in common between $\gamma$ and $\alpha$, and the smoothness of $\alpha$ proves that all these polygonals are in fact the same geodesic.

Asumme that $\alpha$ is not smooth to arrive to a contradiction. Namely assume there is at least one point $q_{i}$ where $\dot{\alpha}_{i}\left(1^{-}\right) \neq \dot{\alpha}_{i+1}\left(0^{+}\right)$. Since $\alpha_{i}(t)=e^{t z^{+}} q_{i} e^{-t z^{+}}$and $\alpha_{i+1}(t)=e^{t z^{-}} q_{i} e^{-t z^{-}}$, then

$$
\Delta \dot{\alpha}_{i}=\dot{\alpha}_{i+1}\left(0^{+}\right)-\dot{\alpha}_{i}\left(1^{-}\right)=\left[z^{+}-z^{-}, q_{i}\right]
$$

is a nonzero vector in $\mathcal{H}_{q}$. We may choose a variation $\gamma_{s}$ of $\alpha=\gamma_{0}$ which is constantly identical to $\alpha$ except in a neighbourhood of $q_{i}$, and such that the variation field $V(t)=$ $\left.\frac{\partial}{\partial s}\right|_{s=0} \gamma_{s}$ equals this vector in $t=1$, namely $V(1)=\Delta \dot{\alpha}_{i}$.

According to the classic first variation formula in a Riemannian manifold (cf. [1] for example),

$$
\left.\frac{d}{d s}\right|_{s=0} L_{2}\left(\gamma_{s}\right)=-\int_{0}^{1}\left\langle V, D_{t} \dot{\gamma}\right\rangle d t-\sum\left\langle V\left(t_{i}\right), \Delta_{i} \dot{\gamma}\right\rangle .
$$


Since $\alpha$ consists of piecewise geodesics and $\alpha$ is also a critical point of the length distance (it is minimizing for the 2 -metric) we obtain

$$
0=\left.\frac{d}{d s}\right|_{s=0} L_{2}\left(\gamma_{s}\right)=\left\|\left[z^{+}-z^{-}, q_{i}\right]\right\|_{2}
$$

which is absurd.

Our main result concerning existence of minimal geodesics follows.

Theorem 5.3. Let $\gamma$ be a smooth curve starting at $\mathrm{p}$ that stays in a neighbourhood of $\mathrm{p}$ of radius $R_{2} / 2$. Then $\gamma$ is longer than the geodesic $\delta$ that joins its endpoints, namely $L_{2}(\gamma) \geq L_{2}(\delta)$.

Proof. We partition $\gamma$ in the same fashion as before, making sure that each piece is shorter than $R_{2}$ in the $\infty$-metric. Now we replace each piece of $\gamma$ with a minimizing geodesic; let us call the breaking points $q_{i}$, where $q_{0}=\mathrm{p}$ and $q_{n}$ is the endpoint of $\gamma$. Clearly the first geodesic is shorter than the first piece of $\gamma$; note that by the triangle inequality, distance between $q_{2}$ and $q_{0}=\mathrm{p}$ is shorter than $R_{2}$, hence we may replace the segments $q_{0} q_{1}, q_{1} q_{2}$ with a shorter geodesic $\delta_{11}$. This geodesic is shorter than the segment of $\gamma$ which joins $q_{0}$ to $q_{2}$, and if we proceed inductively, we end up with a smooth geodesic $\delta$ which joins $q_{0}=\mathrm{p}$ to $q_{n}$, and this geodesic $\delta$ is clearly shorter than $\gamma$.

\section{The tangent and normal bundles}

Let us finish this paper characterizing the tangent spaces of $\mathcal{O}(\mathrm{p})$ as subspaces of the tangent bundle of $\mathcal{P}\left(M_{1}\right)$, and thereon give a characterization of the tangent bundle of $\mathcal{P}\left(M_{1}\right)$ and the normal space of $\mathcal{O}(\mathrm{p})$ at $\mathrm{p}$, using only elements in the algebra $M$. We show in this description that curvature of $\mathcal{O}(\mathrm{p})$ in $\mathcal{P}\left(M_{1}\right)$ is related to the set $\left\{x^{*}=-x \in \operatorname{ker} E: x^{2} \in\right.$ $N\} \subset M$.

Proposition 6.1. Let $q \in \mathcal{O}(\mathrm{p})$, then

$$
(T \mathcal{O}(\mathrm{p}))_{q}=\left\{y \in\left(T \mathcal{P}\left(M_{1}\right)\right)_{q}: E_{1}(y)=0\right\} .
$$

Proof. Note that $\mathcal{O}(\mathrm{p})$ consists of projections $q$ in $M_{1}$ such that $E_{1}(q)=\lambda$ (see [14]). Therefore, if $q(t)$ is a curve in $\mathcal{O}(\mathrm{p})$, then $E_{1}(\dot{q}(t))=0$. In ohter words, $(T \mathcal{O}(\mathrm{p}))_{q} \subset\{y \in$ $\left.\left(T \mathcal{P}\left(M_{1}\right)\right)_{q}: E_{1}(y)=0\right\}$. Conversely, suppose that $y \in\left(T \mathcal{P}\left(M_{1}\right)\right)_{q}$ and $E_{1}(y)=0$. If $q=$ $u \mathrm{p} u^{*}$, by conjugating with $u^{*}$ one may suppose $q=\mathrm{p}$. Then there exists $x \in M_{1}, x^{*}=-x$ such that $y=x \mathrm{p}-\mathrm{p} x$. By the properties of the basic construction, there exists $m \in M$ (a priori not necessarily anti-hermitics) such that $x \mathrm{p}=m \mathrm{p}$. Then $-\mathrm{p} x=(\mathrm{p} x)^{*}=\mathrm{p} m^{*}$. Then

$$
0=E_{1}(y)=E_{1}\left(m \mathrm{p}+\mathrm{p} m^{*}\right)=\lambda m+\lambda m^{*},
$$

i.e. $m \in M_{a h}$, and $y=m \mathrm{p}-\mathrm{p} m \in(T \mathcal{O}(\mathrm{p}))_{\mathrm{p}}$.

Now recall that $(T \mathcal{O}(\mathrm{p}))_{\mathrm{p}}=\left\{z \mathrm{p}-\mathrm{p} z: z^{*}=-z, z \in \operatorname{ker} E\right\}$, where $\operatorname{ker} E=N^{\perp} \subset M$.

Proposition 6.2. We have

$$
\left(T \mathcal{P}\left(M_{1}\right)\right)_{\mathrm{p}}=\left\{x \mathrm{p}+\mathrm{p} x^{*}: x \in N^{\perp}\right\} \text { and }(T \mathcal{O}(\mathrm{p}))_{\mathrm{p}}^{\perp}=\left\{x \mathrm{p}+\mathrm{p} x: x^{*}=x, x \in N^{\perp}\right\} .
$$

In particular $\left(T \mathcal{P}\left(M_{1}\right)\right)_{\mathrm{p}} \simeq N^{\perp} \simeq N_{a h}^{\perp} \oplus N_{h}^{\perp} \simeq(T \mathcal{O}(\mathrm{p}))_{\mathrm{p}} \oplus(T \mathcal{O}(\mathrm{p}))_{\mathrm{p}}^{\perp}$.

The Riemannian exponential map of $\mathcal{P}\left(M_{1}\right)$ is given by

$$
\exp _{\mathrm{p}}^{\mathcal{P}\left(M_{1}\right)}\left(x \mathrm{p}+\mathrm{p} x^{*}\right)=e^{\left(x \mathrm{p}-\mathrm{p} x^{*}\right)} \mathrm{p} e^{-\left(x \mathrm{p}-\mathrm{p} x^{*}\right)} .
$$


In the decomposition relative to Jones' projection $\mathrm{p}$ we have

$$
e^{x \mathrm{p}-\mathrm{p} x^{*}}=\left(\begin{array}{cc}
\left.\cos \left(\sqrt{E\left(|x|^{2}\right.}\right)\right) \mathrm{p} & \left.-\operatorname{sinc}\left(\sqrt{E\left(|x|^{2}\right.}\right)\right) \mathrm{p} x^{*} \\
\left.x \mathrm{p} \operatorname{sinc}\left(\sqrt{E\left(|x|^{2}\right.}\right)\right) & \cos \left(\sqrt{x \mathrm{p} x^{*}}\right)
\end{array}\right) .
$$

where $\operatorname{sinc}(z)=\sin (z) z^{-1}$. Moreover,

$$
e^{\left(x \mathrm{p}-\mathrm{p} x^{*}\right)} \mathrm{p} e^{-\left(x \mathrm{p}-\mathrm{p} x^{*}\right)}=\left(\begin{array}{cc}
\left.\cos ^{2}\left(\sqrt{E\left(|x|^{2}\right.}\right)\right) \mathrm{p} & \left.\operatorname{sinc}\left(2 \sqrt{E\left(|x|^{2}\right.}\right)\right) \mathrm{p} x^{*} \\
\left.x \mathrm{p} \operatorname{sinc}\left(2 \sqrt{E\left(|x|^{2}\right.}\right)\right) & \sin ^{2}\left(\sqrt{x \mathrm{p} x^{*}}\right)
\end{array}\right) \text {. }
$$

The action $\mathrm{p} \mapsto e^{\left(x \mathrm{p}-\mathrm{p} x^{*}\right)} \mathrm{p} e^{-\left(x \mathrm{p}-\mathrm{p} x^{*}\right)}$ is effective if $\|x\|<\pi$.

Proof. Let $v \in\left(T \mathcal{P}\left(M_{1}\right)\right)_{\mathrm{p}}=\left\{w \mathrm{p}-\mathrm{p} w: w \in M_{1}, w\right.$ is $\mathrm{p}-$ codiagonal $\}$. Then we have $v=w \mathrm{p}-\mathrm{p} w=R(w) \mathrm{p}+\mathrm{p} R(w)^{*}$ with $R(w) \in M$. Now $E(R(w)) \mathrm{p}=\mathrm{p} R(w) \mathrm{p}=\mathrm{p} w \mathrm{p}=$ 0 , hence $R(w) \in \operatorname{ker} E=N^{\perp}$. On the other hand, for given $x \mathrm{p}+\mathrm{p} x^{*}$ with $x \in \operatorname{ker} E$, let $w=x \mathrm{p}-\mathrm{p} x^{*}$. Then $w=w \mathrm{p}+\mathrm{p} w(w$ is $\mathrm{p}$-codiagonal $)$ and $w \mathrm{p}-\mathrm{p} w=x \mathrm{p}+\mathrm{p} x^{*}$. The isomorphism and the formula for the Riemannian exponential are now clear from the results on the previous sections.

The formula for the exponential $e^{x \mathrm{p}-\mathrm{p} x}$ can be deduced from the elementary formula

$$
\exp \left(\begin{array}{cc}
0 & -Y^{*} \\
Y & 0
\end{array}\right)=\left(\begin{array}{cc}
\cos |Y| & -\operatorname{sinc}\left|Y^{*}\right| Y \\
Y \operatorname{sinc}\left|Y^{*}\right| & \cos \left|Y^{*}\right|
\end{array}\right)
$$

putting $Y=x \mathrm{p}$ and recalling that $\mathrm{p} x \mathrm{p}=E(x) \mathrm{p}=\mathrm{p} E(x)$.

Note that, if $e^{x \mathrm{p}-\mathrm{p} x^{*}}$ commutes with $\mathrm{p}$, then $\left.\cos ^{2}\left(\sqrt{E\left(|x|^{2}\right.}\right)\right) \mathrm{p}=\mathrm{p}$ (equivalently $\cos ^{2}\left(\sqrt{E\left(|x|^{2}\right)}\right)=1$, since $\left.E\left(|x|^{2}\right) \in N\right)$. This equation proves that $\sigma\left(\sqrt{E\left(|x|^{2}\right)}\right) \subset$ $\{k \pi\}_{k \in \mathbb{Z}}$. Since $\left\|E\left(|x|^{2}\right)\right\| \leq\|x\|^{2}<\pi^{2}$, we obtain $\sigma\left(\sqrt{E\left(|x|^{2}\right)}\right)=\{0\}$. Since $\sqrt{E\left(|x|^{2}\right)}$ is a positive operator, it must be zero. Equivalently, since $E\left(|x|^{2}\right) \geq \lambda|x|^{2}$, we obtain $x=0$.

Theorem 6.3. The geodesic of $\mathcal{P}\left(M_{1}\right)$ given by $\gamma_{x}(t)=e^{t\left(x \mathrm{p}-\mathrm{p} x^{*}\right)} \mathrm{p} e^{-t\left(x \mathrm{p}-\mathrm{p} x^{*}\right)}$ is a geodesic of $\mathcal{O}(\mathrm{p})$ iff $x^{*}=-x$ and $x^{2} \in N$, and in this case we have

$$
\gamma_{x}(t)=e^{t x} \mathrm{p} e^{-t x}=\mathrm{p} \cos ^{2}(t|x|)+u \mathrm{p} u^{*} \sin ^{2}(t|x|)+\frac{1}{2}[u, \mathrm{p}] \sin (2 t|x|) .
$$

where $x=u|x|$, with $u^{*}=-u \in N^{\perp}$ a partial isometry such that $u^{*} u=u u^{*}=-u^{2} \in N$, and $|x|=\sqrt{-x^{2}} \in N$.

Proof. Assume first that $x^{*}=-x$ and $x^{2} \in N$. Let $x=u|x|$ be the polar decomposition of $x$, note that $u^{*}=-u$ commutes with $x$. Then $e^{t x} \mathrm{p}=e^{-i(i x)} \mathrm{p}=\cos (i x)-i \sin (i x)=$ $\cos (|x|)+u \sin (|x|)$, because $(i x)^{2 k}=i^{2 k} x^{2 k}=(-1)^{k} u^{2 k}|x|^{2 k}=|x|^{2 k}$. Likewise, $(i x)^{2 k+1}=u i|x|^{2 k+1}$. On the other hand, from the previous proposition we obtain

$$
e^{x \mathrm{p}-\mathrm{p} x *} \mathrm{p}=e^{x \mathrm{p}+\mathrm{p} x} \mathrm{p}=(\cos |x|+x \operatorname{sinc}|x|) \mathrm{p}=(\cos |x|+u \sin |x|) \mathrm{p},
$$

because $\sqrt{E\left(|x|^{2}\right)}=|x|$ since $|x|^{2} \in N$. Hence $e^{t\left(x \mathrm{p}-\mathrm{p} x^{*}\right)} \mathrm{p}=e^{t x} \mathrm{p}$ in this case. 
Assume now that $\gamma_{x}(t)=e^{t\left(x \mathrm{p}-\mathrm{p} x^{*}\right)} \mathrm{p} e^{-t\left(x \mathrm{p}-\mathrm{p} x^{*}\right)} \mathrm{p}=e^{z} \mathrm{p} e^{-t z} \in \mathcal{O}(\mathrm{p})$ with $x \in N^{\perp}$. Computing the first derivative at $t=0$ and multiplying by $\mathrm{p}$ on the right we obtain $x=z$. Computing the second derivative at $t=0$ we obtain $E\left(x^{2}\right)-x^{2}=0$, hence $x^{2} \in N$.

If $x=u|x|$, we have $u^{*} x=u^{*} u|x|=|x|$. Note that $x^{2} \in N$ iff $|x| \in N$. We can write $u=x h(|x|)$ with $h$ a Borel function ([17] ex. IV.3). This shows that $u \in N^{\perp}$. Since $x^{*}=-x$, we have $u^{*}=-u$, and $u^{*} u=u^{*} x h(|x|)=|x| h(|x|) \in N$. The formula for the geodesic now follows from the proposition above.

Corollary 6.4. If $\lambda=\frac{1}{2}$ (i.e. $[N: M]=2$ ), then $\mathcal{O}(\mathrm{p})$ is a totally geodesic submanifold of $\mathcal{P}\left(M_{1}\right)$.

Proof. If $\lambda=\frac{1}{2}$, the extension $[N: M]$ can be represented with $2 \times 2$ matrices with entries in $N$, namely

$$
M=\left(\begin{array}{cc}
n & \theta\left(n^{\prime}\right) \\
n^{\prime} & n
\end{array}\right) \text { and } \quad N=\left(\begin{array}{cc}
n & 0 \\
0 & n
\end{array}\right)
$$

where $\theta$ is an isomorphism of $N$ of order 2. Then $N^{\perp}=\left(\begin{array}{cc}0 & \theta\left(n^{\prime}\right) \\ n^{\prime} & 0\end{array}\right)$, hence $\left(N^{\perp}\right)^{2} \subset N$.

\section{References}

[1] E. Andruchow, Short Geodesics of Unitaries in the $L^{2}$ Metric, Canad. Math. Bull. 48 (2005) no. 3, 340-534.

[2] E. Andruchow, A. Larotonda, L. Recht and D. Stojanoff, Infinite Dimensional HoMOGENEOUS REDUCTIVE SPACES AND FINITE INDEX CONDITIONAL EXPECTATIONS, Illinois J. Math. 41 (1997) no. 1, 54-76.

[3] E. Andruchow and L. Recht, Grasmannians of a Finite algebra in the Strong OPERATOR TOPOLOGY, Internat. J. Math. 17 (2006) no. 4, 477-491.

[4] E. Andruchow and L. Recht, Geometry of Unitaries in a finite Algebra: VARIATIONAL FORMULAS AND CONVEXITY, preprint.

[5] E. Andruchow and D. Stojanoff, Geometry of Conditional Expectations with Finite IndEX, Int. J. Math. 5 (1994) no. 2, 169-178.

[6] E. Andruchow and D. Stojanoff, Nilpotents in Finite Algebras , Integr. Equ. Oper. Theory 45 (2003) 251-267.

[7] H. Araki, M.B. Smith and L. Smith On the homotopical Significance of the type of von Neumann Algebra factors. Comm. Math. Phys. 22 (1971) 71-88.

[8] G. Corach, H. Porta and L. Recht, The GeOMetry of SPACES of Projections IN $C^{*}$-Algebras, Adv. in Math. 41 (1997) no. 1, 54-76.

[9] V.F.R. Jones, Index for subfactors, Invent. Math. 72 (1983) no. 1, 1-25.

[10] S. Lang, Differential and Riemannian manifolds. Third edition. Graduate Texts in Mathematics 160. Springer-Verlag, New York (1995).

[11] J.M. Lee, Riemannian Geometry. Graduate Texts in Mathematics, Springer-Verlag 176 (1997).

[12] L. Mata-Lorenzo and L. Recht, Infinite Dimensional Homogeneous Reductive SPACES, Reporte 91-11, U.S.B. (1991).

[13] E. Michael, Convex structures and continuous selections. Canadian J. Math. 11 (1959) 556-575. 
[14] M. Pimsner and S. Popa. Entropy and index for Subfactors. Ann. Sci. École Norm. Sup. (4) 19 (1986) no. 1, 57-106.

[15] H. Porta, and L. Recht, Minimality of Geodesics in Grassmann manifolds, Proc. Amer. Math. Soc. 100 (1987) no. 3, 464-466.

[16] S. Popa and M. Takesaki The topological structure of the Unitary and AUTOMORPhism Groups of A FACTOR, Commun. Math. Phys. 155 (1993) 93-101.

[17] M. Reed and B. Simon Functional Analysis, Vol I. Academic Press (1993).

Esteban Andruchow and Gabriel Larotonda

Instituto de Ciencias

Universidad Nacional de General Sarmiento

J. M. Gutierrez 1150

(1613) Los Polvorines

Argentina

e-mails: eandruch@ungs.edu.ar, glaroton@ungs.edu.ar 